\title{
Abordagem cirúrgica dos hemangioblastomas intramedulares
}

\author{
Surgical management of intramedullary hemangioblastomas \\ Manejo quirúrgico de los hemangioblastomas intramedulares
}

\author{
Andrei Fernandes Joaquim ${ }^{1}$ \\ Marcos Juliano dos Santos ${ }^{2}$ \\ Hélder Tedeschi ${ }^{3}$
}

\section{RESUMO}

Objetivo: descrever uma experiência e as técnicas para abordagem destas lesões com ênfase nos aspectos microcirúrgicos. Métodos: foram relatados nove casos consecutivos de pacientes submetidos à ressecção de lesões intramedulares operados entre 2000 e 2008. A escala funcional de McCormick foi usada para avaliar o status funcional dos pacientes Resultados: a idade dos pacientes variou de 13 a 45 anos (média de 27,1 anos). Quatro $(44,4 \%)$ pacientes eram homens e cinco $(55,5 \%)$, mulheres. Dois pacientes tinham a doença de von Hippel-Lindau, com outros hemangioblastomas associados. Conforme a localização, sete $(77,7 \%)$ pacientes tinham lesões na região cervical (dois deles com tumores na transição crânio-cervical) e dois $(22,2 \%)$ tinham lesões na região torácica. Todos os pacientes tiveram ressecção total das lesões, sendo que três apresentaram leve piora clínica no pós-operatório imediato. Após seis meses do procedimento cirúrgico, dois pacientes apresentaram melhora clínica, enquanto sete mantinhamse funcionalmente iguais ao período pré-operatório. Conclusões: o conhecimento anatômico e de técnicas

\section{ABSTRACT}

Objectives: to report an experience and to present a surgical technique to achieve total resection and cure. Methods: nine consecutive cases of intramedullary haemangioblastomas, operated between 2000 and 2008 are presented. The functional scale proposed by McCormick was used to evaluate the patients' neurological status. Results: age at presentation varied from 13 to 45 (average 27.1) years. Four $(44.4 \%)$ patients were male and five (55.5\%), female. Two patients had an associated von Hippel-Lindau disease, with others haemangioblastomas. According to the site of presentation, seven (77.7\%) were localized at the cervical region (including two at the cervico-medullary junction), and two $(22.2 \%)$ at the thoracic level. Total resection was achieved in all cases. Three patients had some functional worsening immediately after surgical procedure. After six months, there were no patients with functional worsening comparing with the preoperative status and two patients had clinical improvement. Conclusions: adequate knowledge of anatomy and the correct use of microsurgical

\section{RESUMEN}

Objetivos: describir una experiencia y las técnicas para abordaje de esas lesiones, enfatizando los aspectos microquirúrgicos. Métodos: fueron relatados nueve casos consecutivos de pacientes sometidos a resección de lesiones intramedulares, operados entre 2000 y 2008. La escala funcional de McCormick fue usada para la evaluación del status funcional de los pacientes Resultados: la edad de los pacientes varió de 13 a 45 anos (media de 27,1). Cuatro $(44,4 \%)$ pacientes eran hombres y cinco $(55,5 \%)$, mujeres. Dos pacientes eran portadores del enfermedad de von Hippel-Lindau, con otros hemangioblastomas asociados. Conforme la localización, siete (77,7\%) pacientes tenían lesiones en la región cervical (dos con tumores en la transición cráneo-cervical) y dos $(22,2 \%)$ tenían lesiones en la región torácica. Todos los pacientes tuvieron resección total de los tumores, siendo que tres tuvieron deterioración clínica leve en el post-operatorio inmediato. Después de seis meses del procedimiento quirúrgico, dos pacientes presentaron mejoras clínicas, mientras seis se mantuvieron funcionalmente iguales al periodo pre-operatorio.

\footnotetext{
Trabalho realizado na disciplina de Neurocirurgia da Universidade Estadual de Campinas - UNICAMP - Campinas (SP), Brasil.

'Pós-graduando (Doutorado) da Universidade Estadual de Campinas - UNICAMP _Campinas (SP), Brasil; Aperfeiçoamento em Cirurgia de Coluna pela Universidade da Virgínia - Charlottesville, EUA

${ }^{2}$ Neurocirurgião do Hospital Sanatorinhos - Itu (SP), Brasil.

${ }^{3}$ Doutor, Professor da Disciplina de Neurocirurgia do Departamento de Neurologia da Universidade Estadual de Campinas - UNICAMP - Campinas (SP), Brasil.
} 
microcirúrgicas adequadas permite a ressecação total destas lesões sem agregar morbidade adicional. techniques allowed total resection of these tumors with minimal morbidity and maximum functional recovery.
Conclusiones: el conocimiento anatómico y de técnicas microquirúrgicas adecuadas permite la resección total de estos tumores sin agregar morbididad adicional.

\section{DESCRIPTORES:}

Hemangioblastoma/cirugía; Neoplasias de la médula espinal/cirugía; Microcirugía/ métodos; Enfermedad de von Hippel-Lindau/diagnóstico
Microcirurgia/métodos; Doença de von HippelLindau/diagnóstico

\section{KEYWORDS:}

Spinal cord neoplasms/ surgery; Microsurgery/ methods; von Hippel-Lindau disease/diagnosis
Hemangioblastoma/ cirurgia; Neoplasias de medula espinhal/cirurgia;

\section{INTRODUÇÃO}

Hemangioblastomas do sistema nervoso central (SNC) são neoplasias vasculares benignas (Grau I, segundo a Organização Mundial da Saúde, OMS) e raras, correspondendo a menos de $3 \%$ dos tumores intramedulares ${ }^{1}$. Podem ser esporádicos, em cerca de 70 a $80 \%$ dos casos, ou associados à doença de von Hippel-Lindau (VHL), doença genética de caráter autossômico dominante em 20 a 30\% dos ca$\operatorname{sos}^{2,3}$. Na síndrome de VHL, além de hemangioblastomas, podem ser encontrados hemangiomas retinianos, tumores e cistos renais e pancreáticos, cistos epididimais, tumores do saco endolinfático e feocromocitomas ${ }^{4}$. A mutação da doença está no gene supressor de tumor no cromossomo 3p26-p25, sendo que perdas alélicas e mutações foram encontradas tanto na síndrome quanto nas formas esporádicas $^{5}$. Hemangioblastomas na doença de VHL são frequentes e múltiplos, e continuam a se desenvolver ao longo da vida do paciente, requerendo contínua vigilância. $\mathrm{O}$ diagnóstico de hemangioblastoma associada à doença de VHL é importante, uma vez que permite detectar precocemente um grande número de lesões associadas, permitindo uma melhor abordagem terapêutica. Pacientes com a doença de VHL tendem a apresentar sinais e sintomas mais precocemente do que pacientes com casos esporádicos. O manejo destes pacientes é mais complicado do que os com lesões isoladas, sendo aceito o tratamento cirúrgico nas lesões sintomáticas ${ }^{2}$.

Neste artigo, relatamos nove casos de hemangioblastomas intramedulares, discutindo a técnica cirúrgica de abordagem destas lesões e a evolução dos casos.

\section{MÉTODOS}

Foram avaliados nove casos de hemangioblastomas intramedulares referidos para tratamento cirúrgico no Hospital das Clínicas da Universidade de Campinas (UNICAMP) pelo autor senior (HT) entre os anos de 2000 e 2008. Todos os pacientes com diagnóstico de hemangioblastomas dessa instituição são investigados clinica e radiologicamente quanto à presença de outros tumores sugestivos da síndrome de VHL. O status neurológico dos pacientes foi avaliado por meio da classificação clínico-funcional de McCormick (Quadro 1) elaborada para os ependimomas intramedulares no periódo pré-operatório e no pós-operatório imediato e tardio (pelo menos seis meses após a cirurgia $)^{6}$. Todos pacientes foram operados sem monitorização eletrofisiológica, não disponível nesse serviço.

Os pacientes foram operados em decúbito ventral. Foram administrados cefalosporina de primeira geração para profilaxia cirúrgica e $10 \mathrm{mg}$ de dexametasona na indução anestésica em todos os casos.

\section{QUADRO 1 - Classificação clínico-funcional de McCormick ${ }^{6}$.}

Grau I: neurologicamente normal; leve déficit focal que não afeta significativamente a

função do membro envolvido; leve espasticidade ou anormalidade de reflexos; marcha normal

Grau II: presença de déficit sensório-motor afetando a função do membro envolvido; ocasiona moderada dificuldade

à marcha; dor ou síndrome disestésica importantes, que prejudicam a qualidade de vida do paciente; funcional e

ambulatorialmente ainda independente

Grau III: déficit neurológico mais importante; requer bengalas/muletas para deambular ou comprometimento significativo

bilateral dos membros superiores; pode ou não ser independente funcionalmente

Grau IV: déficit grave; necessita de cadeira de rodas ou bengalas/muletas, com comprometimento significativo bilateral dos membros superiores; geralmente dependentes funcionalmente 
Todos pacientes foram operados com o auxílio de microscópio cirúrgico. Uma incisão posterior mediana após confirmação radiológica do nível acometido foi realizada, seguida de dissecção subperiostal, até exposição total das lâminas. Uma laminoplastia não expansiva realizada com drill pneumático de alta rotação foi utilizada em todos os casos, após nova confirmação radiológica, expondo-se um nível vertebral acima e um abaixo da lesão, removendo-se as lâminas e os processos espinhosos em bloco. Os dois pacientes com lesões na região crânio-cervical foram submetidos à craniectomia suboccipital com retirada do arco posterior de $\mathrm{C} 1$ e da lâmina de $\mathrm{C} 2$. Nenhum paciente recebeu instrumentação adicional. A fixação do crânio foi utilizada em todos pacientes com lesões cervicais por meio de um fixador rígido de crânio com três pinos (tipo Mayfield ou Sugita). A dura-máter foi incisada medialmente, sendo ancorada com suturas. Sob uso de microscopia, a aracnoide foi aberta e foi iniciada a dissecção com coagulação dos vasos aferentes e posterior dissecção lenta e cautelosa da lesão na medula, deixando, por fim, a coagulação de veias de drenagem. $\mathrm{O}$ uso de cauterização com bipolar deve ser minimizado, para não causar lesão térmica adicional. Além disso, por se tratarem de lesões extremamente vascularizadas, o debulking tumoral deve ser evitado. As lâminas foram refixadas com fios simples de sutura (nylon 0). Casos ilustrativos são apresentados nas Figuras 1 e 2.

\section{RESULTADOS}

A idade dos pacientes variou de 13 a 45 anos (média de $27,1)$. Quatro $(44,4 \%)$ pacientes eram homens e cinco $(55,5 \%)$, mulheres. Dois pacientes tinham a síndrome de VHL, com outros hemangioblastomas associados. Conforme a localização, sete $(77,7 \%)$ pacientes tinham lesões na região cervical (dois deles com tumores na transição crânio-cervical) e dois $(22,2 \%)$ tinham lesões na região torácica. Três pacientes apresentavam lesões císticas associadas, responsáveis por grande parte dos sintomas, dado seu efeito de massa e que facilitam na dissecação microcirúrgica. Todos os pacientes tiveram ressecção total da lesão, sendo que três apresentaram leve piora clínica no pós-operatório imediato. Após seis meses da cirurgia, dois pacientes apresentaram melhora clínica, enquanto sete mantinham-se, funcionalmente, como no período pré-operatório.

\section{DISCUSSÃO}

Melmon e Rosen ${ }^{7}$ desenvolveram critérios clínicos para o diagnóstico da doença de VHL. Conforme estes autores, na falta de história familiar ou de hemangioblastomas retinianos, o diagnóstico da doença de VHL requer (1) presença de pelo menos dois hemangioblastomas no SNC ou na retina; ou (2) um hemangioblastoma e uma das seguintes manifestações: carcinoma renal, feocromocitoma, cistos pancreáticos ou cistoadenoma papilar do epidídimo ${ }^{7}$. Contudo, atualmente, o diagnóstico genético da mutação do gene pode ser extremamente útil e preciso, especialmente

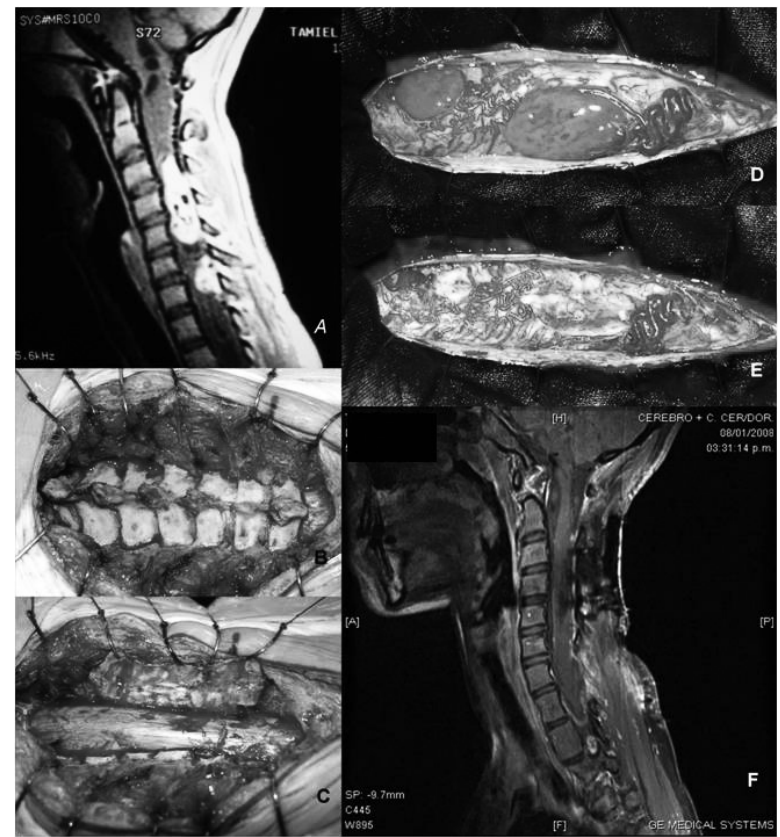

Figura 1

(A) Ressonância magnética pré-operatória da medula cervical. (B) Exposição cirúrgica das lâminas de C2 a T1. (C) Aspecto cirúrgico, com laminotomia open-door - as lâminas estão rebatidas superiormenre. (D) Lesões expostas após abertura da dura-máter. (E) Aspecto do leito cirúrgico após ressecção das lesões. (F) Ressonância magnética pós-operatória: ausência de lesão captante.

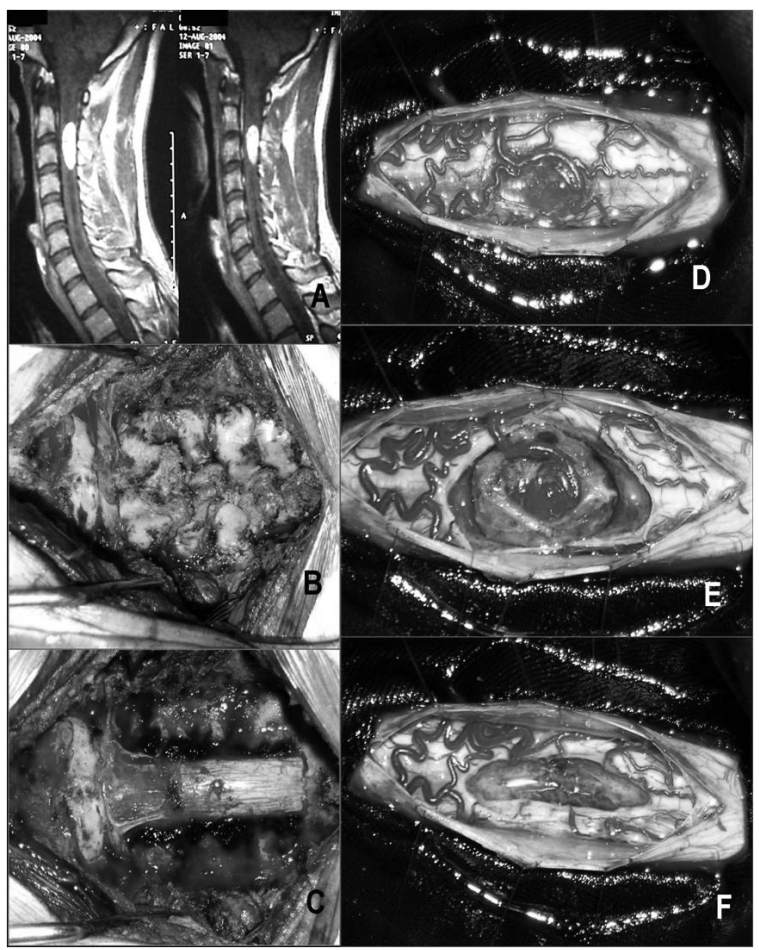

Figura 2

(A) Ressonância magnética pré-operatória. (B) Exposição do occipital até a lâmina de C5. (C) Aspecto cirúrgico pós-laminotomia. (D) Após abertura da dura-máter. (E) Microdissecação da lesão e isolamento da veia de drenagem. (F) Leito operatório após remoção tumoral. 
TABELA 1 - Características dos nove pacientes com hemangioblastomas intramedulares operados.

\begin{tabular}{|c|c|c|c|c|c|c|c|c|}
\hline \multirow[b]{2}{*}{ Caso } & \multirow[b]{2}{*}{ Idade } & \multirow[b]{2}{*}{ Sexo } & \multirow{2}{*}{$\begin{array}{l}\text { Local na } \\
\text { medula }\end{array}$} & \multirow[b]{2}{*}{ Vhl } & \multirow{2}{*}{$\begin{array}{l}\text { Outros locais } \\
\text { acometidos }\end{array}$} & \multicolumn{3}{|c|}{ Grau - McCormick } \\
\hline & & & & & & Pré-operatório & Pós-operatório & $\begin{array}{c}6 \text { meses } \\
\text { pós-operatório }\end{array}$ \\
\hline 1 & 13 & $\mathrm{~F}$ & C & $S$ & C-T-L & III & III & I \\
\hline 2 & 45 & M & C & S & Cerebelo & I & I & I \\
\hline 3 & 23 & $\mathrm{~F}$ & $C^{*}$ & $\mathrm{~N}$ & - & । & $\|$ & । \\
\hline 4 & 24 & $\mathrm{~F}$ & C & $\mathrm{N}$ & - & $\|$ & $\|$ & $\|$ \\
\hline 5 & 39 & M & $\mathrm{T}^{*}$ & $N$ & - & $\|$ & III & ॥ \\
\hline 6 & 20 & $\mathrm{~F}$ & C-B & $\mathrm{N}$ & - & $\|$ & $\|$ & I \\
\hline 7 & 17 & $\mathrm{~F}$ & $C-B^{*}$ & $\mathrm{~N}$ & - & III & III & - \\
\hline 8 & 37 & M & $T$ & $N$ & - & I & $\|$ & I \\
\hline 9 & 26 & M & C & $\mathrm{N}$ & - & $\|$ & $\|$ & $\|$ \\
\hline
\end{tabular}

C: cervical; C-B: cérvico-bulbar; T: torácica; L: lombar; VHL: doença de von Hippel- Lindau; S: sim; N: não.

* cistos associados. Grau segundo: McCormick et al., $1990^{6}$.

em lesões únicas em paciente sem outra manifestação da doença, permitindo vigilância clínica ${ }^{4}$.

O diagnóstico dos hemangioblastomas é dado por exame de ressonância magnética, que mostra lesões nodulares que realçam homogeneamente ao contraste, podendo estar associadas a cistos. Os locais mais acometidos do SNC são, respectivamente, o cerebelo, a medula espinhal e o tronco encefálico, com sintomatologia dependente do tamanho e da topografia da lesão ${ }^{4}$.

A indicação cirúrgica é aceita frente a lesões sintomáti$\operatorname{cas}^{2,8}$, enquanto que o manejo dos tumores assintomáticos é controverso, sendo sugerido, por muitos, a ressecção, quando há evidência de progressão da lesão em exames de imagem seriados, mesmo na ausência de sinais e sintomas ${ }^{9}$. Os sintomas mais comuns são parestesias, paresias e dor axial ou radicular.

A maioria dos hemangioblastomas do tronco e medula espinhal emerge na superfície dorsolateral desses órgãos, o que explica a predominância de sintomas sensitivos no momento do diagnóstico e facilita o acesso cirúrgico.

A remoção efetiva dessas lesões depende de dissecação adequada de plano microcirúrgico entre a superfície tumoral e a medula. Esta dissecação deve ser desenvolvida com a utilização da coagulação bipolar em baixa intensidade na superfície tumoral e tem importância primordial no resultado cirúrgico e na evolução pós-operatória. As artérias nutridoras da lesão são coaguladas e cortadas com microtesouras, conforme a dissecação progride; assim, o tumor perde o turgor e pode ser mais facilmente mobilizado. Não há espaço para debulking tumoral devido à natureza vascular do tumor. Nos casos em que o tumor não acomete a superfície medular, uma mielotomia é a manobra adequada, e o conhecimento anatômico dos tratos e funículos medulares se faz fundamental.

$\mathrm{Na}$ série do presente estudo, dos nove pacientes tratados, somente três evoluíram com discreta piora neurológica no pós-operatório imediato (Tabela 1). Nenhum paciente, em seis meses após o tratamento cirúrgico, se encontrava em condições clínicas piores do que as pré-operatórias. Pelo contrário, dois pacientes, do total de nove, mostraram melhora clínica, comparando-se o status funcional do préoperatório ao encontrado seis meses após o procedimento. Todos os pacientes permaneceram sem recidivas locais das lesões no seguimento pós-operatório. Nenhum paciente recebeu tratamento adjuvante. Atualmente, sabe-se que a quimioterapia possui resultados frustos no tratamento de hemangioblastomas, não devendo ser utilizada ${ }^{10}$. A radioterapia tradicional não deve ser usada devido aos danos adicionais extras causados à medula e, embora com resultados significantes, a radiocirurgia estereotáxica também é inferior ao tratamento cirúrgico adequado ${ }^{11}$.

\section{CONCLUSÕES}

O conhecimento anatômico e de técnicas microcirúrgicas adequadas permitem a ressecção e a cura destas lesões sem agregar morbidade adicional.

\section{REFERÊNCIAS}

1. Kleihues P, Cavenee WK. Pathology and genetics of tumours of the nervous system; Lyon: IARC Press; 2000.

2. Conway JE, Chou D, Clatterbuck RE, Brem H, Long DM, Rigamonti D. Hemangioblastomas of the central nervous system in von Hippel-Lindau syndrome and sporadic disease. Neurosurgery. 2001;48(1):55-63.
3. Neumann HP, Eggert HR, Weigel K, Friedburg H, Wiestler OD, Schollmeyer P. Hemangioblastomas of the central nervous system: A 10-year study with special reference to von Hippel-Lindau syndrome. J Neurosurg. 1989;70(1):24-30.
4. Catapano D, Muscarella LA, Guarnieri V, Zelante L, D'Angelo VA, D'Agruma L. Hemangioblastomas of central nervous system: molecular genetic analysis and clinical management. Neurosurgery. 2005;56(6):1215-21. 
5. Latif F, Tory K, Gnarra J, Yao M, Duh FM, Orcutt ML, et al. Identification of the von Hippel-Lindau disease tumor suppressor gene. Science. 1993;260(5112):1317-20.

6. McCormick PC, Torres R, Post $\mathrm{KD}$, Stein BM. Intramedullary ependymoma of the spinal cord. J Neurosurg. 1990;72(4):523-32.

7. Melmon KL, Rosen SW. Lindau's disease: Review of the literature and study of a large kindred. Am J Med. 1964;36:595-617.

8. Lonser R, Weil R, Wanebo J, DeVroom H, Oldfield E. Surgical management of spinal cord hemangioblastomas in patients with von Hippel-Lindau disease. J Neurosurg. 2003;98(1):106-16.
9. Van Velthoven V, Reinacher PC, Klisch J, Neumann HP, Gläsker S. Treatment of intramedullary hemangioblastomas, with special attention to von HippelLindau disease. Neurosurgery. 2003;53(6):1306-13; discussion 1313-4.

10.Roonprapunt C, Silvera VM, Setton A, Freed D, Epstein FJ, Jallo GI. Surgical management of isolated hemangioblastomas of the spinal cord. Neurosurgery. 49(2):321-7; discussion 327-8.

\section{Correspondência}

Hélder Tedeschi

Rua Mato Grosso, 128, conj.71 Higienópolis

CEP: $01239-040$ - São Paulo (SP), Brasil Fone: (11) 3255-9396

E-mail: hekamarated@mail.com 